\title{
Avaliação da microcirculação da mucosa oral por meio da fluxometria laser doppler em pacientes que recebem quimioterapia e radioterapia
}

\section{RESUMO}

O objetivo deste estudo foi identificar as alterações presentes na microcirculação da mucosa oral por meio da Fluxometria Laser Doppler (FLD) em pacientes que recebem quimioterapia (QT) e/ou radioterapia (RT) com alto risco de desenvolver a mucosite oral (MO) e relacionar o fluxo sanguíneo com a graduação da $\mathrm{MO}$ e o fluxo sanguíneo com a graduação de dor. A amostra foi composta por indivíduos sob quimioterapia com alto risco de desenvolver $\mathrm{MO}(\mathrm{GQ})$; indivíduos sob RT acima de 20Gy em região de cabeça e pescoço - concomitante ou não a QT (GR) e indivíduos sem doença oncológica (GC). Foi realizada a mensuração do fluxo sanguíneo na região geniana através da FLD, sendo que no GQ foram realizadas 7 avaliações, no GR foram 8 avaliações e no GC apenas 1 avaliação; a dor em mucosa oral foi avaliada através da Escala Visual Analógica (EVA) e a MO através das escalas da Organização Mundial de Saúde (MO-OMS) e da Oral Mucositis Assessment Scale (MO-OMAS). O GQ foi composto por 7 indivíduos, o GR por 10 indivíduos e o GC por 12 indivíduos. Observamos que no GQ ao compararmos a evolução da MO-OMS ao longo do tempo com o momento inicial (T0) as manifestações são piores nos momentos T2, T3, T4. No GR também observamos a piora da manifestação da MO-OMS nos momentos T3, T4, T5, T6 com recebimento de dose de radiação entre 22 à 60Gy. Na MO-OMAS observamos que no $\mathrm{GQ}$ as piores manifestações ocorreram nos momentos T2, T3, T4, quando comparadas ao momento inicial (T0). No GR as piores manifestações da MO-OMAS foram coincidentes com a da MO-OMS no GR nos momentos T3, T4, T5, T6 e em dose de radiação entre 22 à $60 \mathrm{~Gy}$. Em relação ao fluxo sanguíneo observamos que no $G Q$ houve aumento do fluxo sanguíneo ao longo do tempo, nos momentos $\mathrm{T} 1, \mathrm{~T} 2, \mathrm{~T} 4, \mathrm{~T} 5$ quando comparamos ao momento inicial (T0), com exceção do momento T3 onde observou-se diminuição no fluxo sanguíneo. No GR também houve aumento do fluxo sanguíneo nos momentos T1, T2, T3, T5, T6 quando comparamos com o momento inicial (T0), com exceção do T4 (dose de radiação entre 32 a 40Gy), onde observou-se diminuição no fluxo sanguíneo. Concluímos que as alterações no fluxo sanguíneo da região geniana de indivíduos submetidos a QR e RT foram identificadas pela FLD e permitiu avaliar que as piores manifestações de MOOMS, MO-OMAS e pior intensidade da dor estão relacionadas com menor fluxo sanguíneo.

Palavras Chaves: Mucosite oral. Fluxometria laser doppler. Microcirculação. 